\title{
A new prescription for the mass-loss rates of hydrogen-free WR stars
}

\author{
Frank Tramper ${ }^{1}$, Hugues Sana ${ }^{2}$ and Alex de Koter ${ }^{2,3}$ \\ ${ }^{1}$ European Space Astronomy Centre (ESA/ESAC), Operations Department, \\ Villanueva de la Cañada (Madrid), Spain \\ email: ftramper@sciops.esa.int \\ ${ }^{2}$ Institute of Astrophysics, KU Leuven, Celestijnenlaan 200 D, B-3001, Leuven, Belgium \\ ${ }^{3}$ Anton Pannekoek Institute for Astronomy, University of Amsterdam, P.O. Box 94249, \\ 1090 GE Amsterdam, The Netherlands
}

\begin{abstract}
We present a new empirical prescription for the mass-loss rates of hydrogen-free WolfRayet stars based on results of detailed spectral analyses of WC and WO stars. Compared to the prescription of Nugis \& Lamers (2000), $\dot{M}$ is less sensitive to the surface helium abundance, implying a stronger mass loss at the late stages of Wolf-Rayet evolution. The winds of hydrogenfree WN stars have a strong metallicity dependence, while those of WC and WO stars have a very weak metallicity dependence.
\end{abstract}

Keywords. stars: Wolf-Rayet, stars: mass loss, stars: winds, outflows

Wolf-Rayet (WR) stars are characterised by emission-line spectra as a result of their very strong stellar winds. The amount of mass lost in the WR phase strongly impacts the final surface abundances (and hence the supernova type), as well as the nature of the compact remnant left behind. The current standard prescription from Nugis \& Lamers (2000) works well for WN and WC stars, but cannot reproduce the mass-loss rates of WO stars (Tramper et al. 2015).

Here we present an improved prescription for the mass-loss rates of hydrogen-free WN, WC, and WO stars. Details of the derivation of the prescription and a discussion on its dependences are given in Tramper et al. (2016). The mass-loss prescription with $1 \sigma$ uncertainties on the coefficients is

$$
\log \dot{M}=-9.20( \pm 0.35)+0.85( \pm 0.06) \log \frac{L}{L_{\odot}}+0.44( \pm 0.08) \log Y+\alpha \log \frac{Z_{\text {ini }}}{Z_{\odot}},
$$

where $\alpha=0.25( \pm 0.08)$ for WC and WO stars and $\alpha=1.3( \pm 0.2)$ for hydrogen-free WN stars. The relation provides mass-loss rates with an uncertainty of $\sigma=0.06$ dex for WC and WO stars, and $\sigma=0.2$ dex for hydrogen-free WN stars.

Compared to Nugis \& Lamers (2000), WR stars have a higher mass loss towards the end of their evolution, when the surface helium abundance is low, and at low metallicities if the WC stage can be reached. There is a strong change in metallicity dependence from the WN to the WC states, implying that carbon driving may be relatively important. Implications of the new prescription include lower final masses, higher final surface abundances of carbon and oxygen, and a longer duration of the WC and WO stages.

\section{References}

T. Nugis \& H. J. G. L. M. Lamers 2000, $A \& A$, 360, 227

F. Tramper, S. M. Straal, D. Sanyal, et al. 2015, A\&A, 581, 110

F. Tramper, H. Sana, A. de Koter 2016, ApJ, 833, 133 